\title{
Abstracts From ASENT 2004 Annual Meeting March 11-13, 2004
}

\section{Multiple Imputation Compared with Conventional Methods for Analysis of Categorical Repeated Measures

\author{
Yuyan Duan,* Ilya Lipkovich, ${ }^{\dagger}$ and Saeeduddin Ahmed $^{\dagger}$ \\ *Virginia Polytechnic Institute and State University, \\ Blacksburg, Virginia, ${ }^{\dagger}$ Lilly Research Laboratories, \\ Indianapolis, Indiana
}

Background: Analyses of categorical repeated measures of clinical data using conventional methods can give biased estimates of treatment effects and associated SEs when dropouts are not completely at random (depending on observed clinical outcomes). We test the utility of multiple imputation (MI) analysis in reducing these biases.

Methods: We used simulation to compare performance of MI versus conventional methods, including restricted pseudolikelihood methods and generalized estimating equations, in five typical clinical profiles for 1) estimating overall treatment effects, and 2) treatment differences at last scheduled visit.

Results: The power to detect treatment differences with MI is consistently higher than with conventional methods. Type I error rates (estimated from scenarios in which no treatment difference existed) were consistently smaller with MI than with conventional methods. However, MI tended to overestimate variability of treatment differences at endpoint. Among tested profiles, the advantage of MI over conventional methods in terms of power to detect overall treatment differences was greatest when treatments separated from each other early, then converged later.

Conclusion: Compared to conventional techniques, MI may lead to less biased estimates of treatment differences in categorical analyses of continuous data, especially in clinical trials with a high (40-60\%) proportion of dropouts. However, MI did not perform well when dropouts were (partially) driven by clinical outcomes that were also not observed. Of course, this conclusion is limited by the specifics of the simulation scenarios tested and as such, does not constitute theoretical proof.

This work was supported by Eli Lilly and Company.

\section{Early Weight Gain as a Predictor of Substantial Weight: Results from Seven Studies of Olanzapine for the Treatment of Bipolar Disorder}

Saeeduddin Ahmed, Ilya Lipkovich, Jonna Ahl, Thomas Hardy, Diane Haldane, Robert Baker, and Mauricio Tohen

Background: Atypical antipsychotics are being used as mood stabilizers for treatment of bipolar disorder. Weight gain, sometimes substantial, is observed during treatment with some of the atypical agents.

Objective: To examine early weight gain (EWG) during olanzapine (OLZ) treatment as a predictor of substantial weight gain (SWG) later in treatment.

Methods: Data were pooled from seven randomized, multicenter studies in bipolar patients $(n=2231$ at initiation of olanzapine). Endpoint for this analysis was 32 weeks ( $n=$ 518). SWG was defined as gaining at least $10 \mathrm{~kg}$ at endpoint. Other definitions of SWG (5 and $7 \mathrm{~kg}$ ) were also evaluated. EWG was determined as the weight gain threshold that optimized discrimination of patients with SWG.

Results: At an EWG threshold of about $2 \mathrm{~kg}$ in the first 3 weeks, among patients with SWG $61 \%$ also had EWG (sensitivity), and $69 \%$ of patients without SWG did not have EWG (specificity). Among those patients who did not have EWG, $83 \%$ did not have SWG. Addition of baseline characteristics (including age, gender, baseline body mass index, nonwhite race) moderately improved predictability. Results for other SWG cut-offs were qualitatively similar.

Conclusions: EWG of about $2 \mathrm{~kg}$ during the first 3 weeks of treatment with OLZ predicted later SWG. Patients with lesser EWG appeared much less likely to have SWG. Further research in larger patient populations and for longer time periods is necessary to explore the predictive power of EWG for SWG with olanzapine as well as other medications, particularly in patients with symptomatic bipolar disorder.

This work was supported by Eli Lilly and Company.

\section{Predominance of Psychiatric-Based Reasons for Antipsychotic Treatment Discontinuation}

\section{Hong Liu-Seifert, Kristine Healey, and Bruce J. Kinon}

Introduction: This research was conducted to better understand the phenomenon of antipsychotic treatment discontinuation and to provide insight into overall treatment effectiveness.

Methods: This was a post hoc, pooled analysis based on four randomized, double-blind clinical trials that had a duration of 24-28 weeks. The four studies included 822 olanzapine-treated patients and 805 patients treated with risperidone, quetiapine, or ziprasidone.

Results: Adverse events related to worsening in psychiatric symptoms accounted for 50\% (100 of 200) of all adverse events leading to treatment dropouts, with worsening in psychotic disorder (35 of 200) and suicide (completed/attempt/ideation, 18 of 200) being the most frequent events. Most decisions of discontinuation due to lack of efficacy were made based on patient perception (132 of 164). Clinical rating scales showed patients who discontinued because of lack of efficacy received no relief of their psychiatric symptoms.

Conclusions: This research demonstrated that worsening of underlying psychiatric symptoms as well as patients' perception of their failure to improve overwhelmingly contribute to treatment discontinuation, which can threaten patient wellbeing with the morbid consequences of illness exacerbation. Better understanding of what causes discontinuation may provide a strategy to improve patient engagement in long-term therapy and to increase patient access in realizing the goals of an effective treatment.

This work was supported by Eli Lilly and Company. 


\section{Comparison of Olanzapine and Lithium Treatment Groups Based on Lithium Blood Levels: A 52-Week Bipolar Relapse Prevention Study}

Saeed Ahmed, Yuyan Duan, Kristine Healey, Ilya A. Lipkovich, and Mauricio F. Tohen

Purpose: Lithium is considered a standard treatment for prophylaxis in bipolar disorder. Appropriate blood lithium levels for maintenance have been reported to be between 0.8 and $1.0 \mathrm{~mm}$. Efficacy of olanzapine versus lithium subgroups, based on blood levels, has previously not been reported.

Methods: This 52-week, randomized, parallel, double-blind study compared bipolar relapse on flexibly dosed lithium or olanzapine. For this analysis, we defined two post hoc lithium subgroups. Lower level group (LLG) comprising subjects with mean lithium levels $\leq 0.8 \mathrm{mEq} / \mathrm{l}$ and higher level group (HLG) comprising subjects with mean lithium levels $>0.8 \mathrm{mEq} / \mathrm{l}$.

Results: The olanzapine treatment group had a greater time to relapse into mania or depression (YMRS total score $>15$ and/or HAMD-21 total score $>15$ and/or hospitalization for mania or depression) compared to the HLG $(p=0.014)$ but not to the LLG. The olanzapine treatment group had lower rates of manic relapse based on hospitalization for relapse and/or symptomatic rating scale criteria than both lithium treatment groups (olanzapine $14.7 \%$ vs LLG 30.5\%; $p=0.002$ and HLG 32.9\%; $p<0.001$ ).

Conclusion: The olanzapine-treated patients had statistically significant lower rates of manic relapse than lithium-treated patients, irrespective of lithium level.

This work was supported by Eli Lilly and Company.

\section{Patterns of Weight Change in Patients with Bipolar Disorder During Treatment with Olanzapine}

\author{
Saeeduddin Ahmed,* Ilya Lipkovich,* \\ Thomas Hardy,* Vicki Hoffmann,* \\ Dong Ding,* Mauricio Tohen,* \\ and Ellen Frank ${ }^{\dagger}$ \\ * Lilly Research Laboratories, Indianapolis, \\ Indiana $;^{\dagger}$ Department of Psychiatry, University of Pittsburgh,
} Pittsburgh, Pennsylvania

Background: Olanzapine is an atypical antipsychotic and mood stabilizing agent associated with weight gain. Weight change patterns in patients with schizophrenia have previously been reported.

Objective: In this post hoc analysis, we examine patterns of weight gain in patients with bipolar disorder during treatment with olanzapine.

Methods: Seven clinical trials were included in this analysis. These trials included patients who suffered from an acute or index manic, depressed, or mixed episode. In four clinical trials, olanzapine was administered as a monotherapeutic agent. In three other studies, psychotropic agents were administered with olanzapine. Data were analyzed by last observation carried forward, observed cases, and mixed models repeated measures analyses.

Results: Mean weight gain in patients with bipolar disorder in the seven studies included in this analysis was $3.93 \mathrm{~kg}$ by last observation carried forward (LOCF) analysis, and $5.98 \mathrm{~kg}$ by observed case (OC) analysis. By three analytic methods (LOCF, OC, and mixed model repeated measures), it was apparent that in all studies, the rate of weight gain was greatest in the first weeks of treatment, and gradually diminished after
4-16 weeks. Similarly, the probability of gaining $7 \%$ of initial body weight was highest in the first weeks of treatment.

Conclusions: Patterns of weight gain associated with olanzapine are similar in patients with schizophrenia and bipolar disorder. Weight gain patterns were also similar in patients who were on olanzapine alone and on olanzapine and other psychotropic medications.

This work was supported by Eli Lilly and Company.

\section{Correlations Between Clinician-Rated Cognition and Patient-Rated Health State in a Schizophrenia Obs}

Lizheng Shi, Janey Shin, Saeed Ahmed, Diego Novick, Paul Berg, Haya Ascher-Svanum, Josep Maria Haro, Isabelle Gasquet, and Spyridon Tziveleskis

Background and Objectives: Impairments in multiple domains of cognition in patients with schizophrenia are important determinants of functional deficits. Comprehensive assessment of cognition entails administration of performance-based neuropsychological instruments, which is often difficult in clinical practice settings. This study examined the correlations between a new clinician-rated measure [clinical global impression rating for cognition (CGI-cog)] and a patient-rated overall health state on the EuroQol-5D (EQ-5D).

Methods: We analyzed 1-year data from the 3-year prospective noninterventional multinational European Schizophrenia Outpatient Health Outcomes (SOHO) study of ambulatory patients with schizophrenia $(n=10,972)$, involving 1096 psychiatrists in routine practice settings in 10 European countries, which evaluates a variety of health outcomes and associated direct healthcare costs. Spearman correlations (for CGI-cog with EQ-5D at the next visit) were used to explore the association between CGI-cog and EQ-5D scale in patients who were at most moderately psychotic at baseline $(n=6572)$. The EQ-5D scale provides a composite score based on five dimensions, and a separate score from a visual analog scale (EQVAS).

Results: At baseline, 3, 6, and 12 months, the EQ-5D composite and EQVAS scores were consistently correlated with CGIcog to a greater extent than with global clinical scores of psychotic symptoms. The correlations for CGI-cog at visit 1 and EQVAS at visit 2 was -0.20 , CGI-cog at visit 2 and EQVAS at visit 3 was -0.29 , and CGI-cog at visit 3 and EQVAS at visit 4 was -0.32 (all $p<0.0001$ ). Similar correlations were observed between CGI-cog and the EQ-5D composite score. High CGI-cog scores indicate severe symptoms of cognition and low EQVAS scores indicate a lower health state. Therefore, these negative correlations suggest greater cognitive deficits are associated with poorer health status and quality of life.

Conclusions: Although clinician-rated measures of cognition cannot substitute for performance-based assessments of cognition, the CGI-cog appears to modestly correlate with a measure of overall health state to a greater extent than do global ratings of positive psychotic symptoms. Correlations between CGI-cog and each functional outcome appear to be temporal which is indicative of causation. Further research is necessary to explore relationship of CGI-cog with functional and neuropsychological measures.

L.S., J.S., S.A., D.N., P.B., and H.A.-S. are full-time employees and shareholders with Eli Lilly and Company. J.M.H. and I.G. are the advisors for the SOHO study, which is sponsored by Eli Lilly and Company. S.T. is a contract health economist with Eli Lilly and Company. 


\section{Neuroprotective Effect of Rasagiline in a Rodent Model of Parkinson's Disease}

Fabio Blandini,* Marie Therese Armentero,* Roberto Fancellu, ${ }^{* \dagger}$ and Giuseppe Nappi**

*Laboratory of Functional Neurochemistry, Neurological Institute “C. Mondino," Pavia, Italy; ${ }^{\dagger}$ University of Insubria, Varese, Italy; ${ }^{\ddagger}$ Department of Neurology and

Otorhinolaryngology, University of Rome "La Sapienza," Rome, Italy

Monoamine oxidase type B (MAO-B) inhibitors of the propargylamine family have been extensively investigated for their potential neuroprotective properties, particularly for Parkinson's disease (PD). The prototype of this class of compounds, selegiline, has shown antioxidant and neuroprotective effects in experimental studies, although the neuroprotective activity in PD patients has remained controversial. Recently, considerable attention has been attracted by rasagiline $[\mathrm{R}(+)-$ $N$-propargyl-1-aminoindane], a potent and selective propargylamine, which is structurally related to, yet different from, selegiline in that, unlike this latter, is not metabolized to amphetamine and/or methamphetamine. In vitro studies have proposed neuroprotective properties for rasagiline, which would be related to the intrinsic activity of the drug, rather than to MAO-B inhibition. Neuroprotection by rasagiline has been substantially confirmed in vivo, although relatively few data are available on the ability of rasagiline to block substantia nigra compacta $(\mathrm{SNc})$ degeneration in animal models of $\mathrm{PD}$, particularly after chronic administration. Therefore, the aim of our study was to explore the neuroprotective potential of chronic rasagiline administration in a rodent model of $\mathrm{PD}$, based on the intrastriatal injection of 6-hydroxydopamine (6-OHDA), a procedure that causes a discrete, progressively evolving lesion of the nigrostriatal pathway. One hour after receiving a unilateral injection of 6-OHDA into the striatum, Sprague Dawley rats began a 6 -week treatment with rasagiline $(0.8$ or $2.5 \mathrm{mg} /$ day, s.c.) or saline (controls). Animals were tested at the fourth week for the presence of rotational behavior after systemic administration of apomorphine, and at the sixth week for survival of $\mathrm{SNc}$ dopaminergic neurons. Results show that both doses of rasagiline markedly increased survival of dopaminergic neurons (as expressed by the $\mathrm{n}$. of tyrosine hydroxylase-positive cells) in the lesioned SNc, compared to controls (+97\% and $+119 \%$, respectively). A protective effect, although of lesser magnitude, was also observed at the level of the nigrostriatal terminals, within the injected striatum. Curiously, only the lower dose of rasagiline abolished the rotational behavior; this may be related to a dose-dependent enhancement of apomorphine efficacy, which may have counteracted the reduction of the motor stereotypy. In conclusion, our study supports the neuroprotective potential of chronic rasagiline administration in an experimental model of PD.

This work was supported by Teva Pharmaceutical Industries Ltd. (Netanya, Israel).

\section{Novel Fc Receptor Ligands as Treatment for CNS Inflammatory Diseases: A Potential Replacement}

\author{
David White, Mark Jensen, and Barry Arnason \\ Department of Neurology, The University of Chicago, \\ Chicago, Illinois
}

The goal of the present study was to design recombinant proteins that can replace many of the therapeutic activities of intravenous immunoglobulin (IVIG). IVIG is successfully used as a therapy for an expanding number of neuroinflammatory processes, including autoimmune syndromes. Yet, IVIG is expensive and its use is limited by chronically short supplies. The immunomodulatory activity of IVIG results, in part, from ligation of $\mathrm{Fc}$ receptors (FcRs) expressed on innate immune system cells. We have designed and expressed a novel class of $\mathrm{Fc}$ receptor ligands (FcRLs) that mimic key actions of IVIG with respect to their interactions with FcRs and may represent an alternative to IVIG for therapeutic interventions. FcRLs are linear polymers of a hinge- $\mathrm{CH} 2 \mathrm{U}$ derived from $\mathrm{IgG}$. The hinge- $\mathrm{CH} 2 \mathrm{U}$ encompasses the binding region for $\mathrm{Fc}$ receptors. We have tested FcRLs containing 2, 3, and 4 hinge-CH2 U (R2, $\mathrm{R} 3$, and R4) for their ability to control cellular function in comparison to the $\mathrm{Fc}$ region from IgG alone (R0). In an FcRdependent activation assay using peripheral blood mononuclear cells, proliferative response correlates directly with polymer length. Direct comparison reveals that FcRL-R3 and FcRL-R4 are over 100-fold more potent than either an IVIG preparation or the anti-FcR monoclonal antibody $3 \mathrm{G} 8$. FcRL-R4 profoundly reduces the production of monocyte-derived IL-12p40 in an in vitro[r] assay using either human or murine monocytes. As IL-12p40 production is critical for the induction of autoimmunity in several animal models and is increased in multiple sclerosis (MS), we determined whether FcRL-R4 would impact the severity or onset of experimental allergic encephalomyelitis (EAE) in the SJL/J mouse, a rodent model for MS. EAE was induced by immunization with a proteolipid protein (PLP) peptide. Intravenous FcRL-R4, given at time of immunization, greatly reduces disease activity during both the acute and recurrent phases of the disease. Intravenous FcRL-R4, given after disease onset, promptly reduces disease activity. The data indicate that FcRLs can suppress initiation and progression of disease in the PLP-EAE model both before disease onset and during times of active disease. FcRLs may represent a novel strategy for treatment of CNS inflammatory diseases, including MS.

\section{European and American Perspectives on the Use of Sham Surgery in Parkinson's Disease Research}

\author{
Samuel Frank,* Karl Kieburtz,* Robert Holloway,* \\ Renee Wilson,* Carol Zimmerman,* and Scott Kim ${ }^{\dagger}$ \\ *Department of Neurology, University of Rochester, \\ Rochester, New York; ${ }^{\dagger}$ Psychiatry and Bioethics Program, \\ University of Michigan, Ann Arbor, Michigan
}

Introduction: The use of sham surgery in clinical trials for Parkinson's disease (PD) is controversial. We assessed and compared the views of basic and clinical researchers from the United States and Europe regarding sham surgery as a control condition in a hypothetical gene transfer neurosurgical trial for PD.

Procedures: We distributed the survey at the annual meeting of the Huntington Study Group (HSG) and at the Network of European CNS Transplantation and Restoration (NECTAR). The three-page survey assessed researchers' views regarding the following: prior probability of efficacy and safety of the proposed gene transfer intervention; the ethical and scientific merits of blinded (sham surgery) versus open control condition and whether the burdens of sham surgery condition are justified by the potential benefits; and vulnerability of PD research subjects.

Results: The response rates were 59 of 230 (26\%) for HSG and 39 of $68(57 \%)$ for NECTAR. Most HSG respondents were neurologists (90\%); most NECTAR respondents were neuroscientists $(75 \%)$. The NECTAR group was more optimistic about the probable efficacy of the intervention (46\% vs $31.3 \%$, 
$p<0.001$ ). $90 \%$ of HSG respondents believed the risk of sham surgery is justified by the potential benefits to science and to society versus $53 \%$ for NECTAR respondents. $31 \%$ of NECTAR respondents believed PD subjects are too vulnerable to offer randomized controlled trial involving sham surgery to them, versus $9 \%$ of HSG respondents. In univariate analyses, location of meeting and professional roles predicted responses; in multivariate analysis, only location of meeting was a significant predictor, with HSG respondents much more likely to favor sham surgery (odds ratio, 54; $95 \%$ confidence interval $4-663 ; p<0.001)$

Conclusions: Although the majority of surveyed researchers believe sham surgery control is scientifically and ethically justified, European respondents (and perhaps basic scientists more than clinical scientists) are more opposed to sham surgery controls in PD surgical trials. The ethics and science of sham surgery controls in PD trials deserve further discussion in the field.

\section{In Search of a Short-Term Outcome for Parkinson's Disease Futility Studies}

Jordan J. Elm, Barbara C. Tilley, Yuko Y. Palesch, Paulo Guimaraes, Christopher Goetz, Bernard Ravina, and Karl Keiburtz

Objective: To determine the most suitable outcome measure for a futility study in Parkinson's disease (PD) using several historical PD data sets.

Background: Futility studies are designed to test new treatments in a short period of time in a small number of patients and determine if they are worthy of larger and/or longer term studies or should be abandoned. The single best outcome measure most sensitive to tracking disease progression in the shortterm, however, has not been determined.

Methods: The authors accessed data sets from one clinical series and five clinical trials and compared scale items, subscores, and total scores on the UPDRS, Schwab and England, and Hoehn and Yahr scales. Descriptive statistics (such as box plots) and paired $t$ tests (of change from baseline to 6,12, and 18 months) were used to examine standard clinical rating scales outcome patterns. Survival outcome measures were developed and explored with Kaplan-Meier curves.

Results: Patients who did not require symptomatic therapy at baseline were the only sample among those studies for which any outcome measure showed a statistically significant worsening from baseline. Among de novo patients, total UPDRS change required the smallest sample size for all the outcome measures explored.

Conclusions: Of those outcome measures explored and developed, total UPDRS (parts 1, 2, 3) is the most responsive measure for detection of short-term change (12 months) in de novo PD subjects. This design is being applied to the National Institute of Neurological Disorders and Stroke-sponsored Neuroprotection Exploratory Trials in PD.

This work was supported by National Institutes of Health/ National Institute of Neurological Disorders and Stroke Grant U01NS043127.

\section{Levotofisopam: A Novel Homophthalazine with Potential for Treatment of Stress-Related Disorders}

Steven M. Leventer, Karen Raudibaugh, John C. Keogh, Robert F. Kucharik, Deirdre O'Hara, Naidong Ye, Kimm Galbraith, Brian Speicher, and Kevin L. Keim
Introduction: Levotofisopam is the S-enantiomer of racemic tofisopam, a homophthalazine prescribed outside the United States for such stress-related disorders as anxiety, functional gastrointestinal disorders, and symptoms of menopause. Thought to affect autonomic tone via interaction with subcortical 2,3-benzodiazepine receptors, levotofisopam has shown promise in animal models of stress-related gastrointestinal dysfunction and menopause. We conducted a trial of levotofisopam in healthy human volunteers.

Methods: The safety, tolerability, and pharmacokinetics of ascending single doses of levotofisopam were examined in a randomized, double-blind, placebo-controlled trial in healthy human volunteers. A total of 42 subjects were randomized to receive single doses of levotofisopam $(50 \mathrm{mg}, 100 \mathrm{mg}, 200 \mathrm{mg}$, $300 \mathrm{mg}$, or $400 \mathrm{mg} ; n=6 /$ dose) or placebo $(n=12)$.

Results: Levotofisopam was generally well tolerated up to the highest dose level (400 mg). The incidences of treatmentemergent adverse events (TEAEs) were similar for levotofisopam $(40 \%)$ and placebo (50\%). All TEAEs in levotofisopamtreated subjects were mild, and there were no clear drug or dose relationships for any TEAE. No serious or severe TEAEs occurred during the study, no subjects discontinued the study prematurely due to TEAEs, and no concomitant medications were required for treatment of any TEAE. Levotofisopam reached $\mathrm{C}_{\max }$ approximately $1 \mathrm{~h}$ after administration at all doses, with mean $\mathrm{C}_{\max }$ ranging from 64 to $1201 \mathrm{ng} / \mathrm{mL}$ and mean area under the curve (AUC) from 87 to $2924 \mathrm{ng} \cdot \mathrm{hr} / \mathrm{mL}$. The mean half-life of levotofisopam appeared to lengthen as the dose increased, from $1.4 \mathrm{~h}$ at $50 \mathrm{mg}$ to $8.1 \mathrm{~h}$ at $400 \mathrm{mg}$. The disproportionate increases in $\mathrm{C}_{\max }$ and AUC relative to increasing dose suggest that the bioavailability of levotofisopam may be somewhat dose-dependent.

Conclusions: Levotofisopam appears to be well tolerated at single doses of up to $400 \mathrm{mg}$ in healthy volunteers. This finding, combined with robust responses in preclinical studies, support additional clinical study of levotofisopam in the treatment of stress-related conditions, such as anxiety and the symptoms of menopause. A multiple-dose study of the effects of levotofisopam in healthy men and menopausal women is currently underway.

This work was supported by Vela Pharmaceuticals Inc.

\section{Central Cholinergic Augmentation Improves Reaction Time But Not Recognition Memory in Young Healthy Adults}

\author{
Alireza Atri, Matthew L. Lopresti, \\ Seth J. Sherman, Haline E. Schendan, \\ Michael E. Hasselmo, and Chantal E. Stern
}

Introduction: The cholinergic hypothesis served as a basis for the development of acetylcholinesterase inhibitors (ACHEIs) and their current use in the treatment of Alzheimer's disease (AD). It is unclear whether augmenting cholinergic modulation by oral ACHE-I affects cognitive processes in healthy young adults (HYA).

Methods: Seventeen HYA were given $8 \mathrm{mg}$ PO galantamine hydrobromide, an ACHE-I and nonpotentiating ligand of the nicotinic ACH-receptor, or placebo, in a double-blind design, to study the effects of central cholinergic augmentation on an episodic visual item recognition paradigm and a serial reaction time task (SRTT). Subjects studied complex visual images and on a subsequent recognition test their memory confidence ratings and reaction times (RTs) were recorded. Subjects then performed a SRTT where their RTs to cued key presses at one 
of four locations were recorded during presentation of blocks of random locations interspersed with blocks of second-order conditional sequence locations, first without (implicit condition) and then with (explicit condition) the knowledge that a repeating sequence of locations existed.

Results: The galantamine group exhibited significantly lower RTs compared to the placebo group. Recognition memory performance was not improved by galantamine. A subgroup $(n=3)$ of subjects receiving galantamine exhibited recognition impairment despite normal performance on a visual discrimination task and the SRTT. Galantamine and placebo groups showed progressively significantly lower RTs over trials, demonstrating RT learning effects, and lower RTs during explicit compared to implicit blocks.

Conclusions: These preliminary results support that central cholinergic augmentation by ACHE-Is in YHA improves cognitive processing speed as reflected by a lowering of RTs during performance of cognitive tasks. This is consistent with findings in patients with $\mathrm{AD}$ given ACHE-Is and in YHA injected with physostigmine. In YHA, galantamine does not improve episodic visual item recognition performance, and may potentially impair performance in some. Together, these suggest a dissociable effect of galantamine on cognitive processes in YHA. Heterogeneity in regional and baseline cholinergic levels and a differential effect of ACHE-Is on cognitive processing speed and attentional networks compared to mnemonic networks may serve as a partial explanation for the heterogeneity and pattern of the clinically observed effects of ACHE-Is on cognition and function in patients with AD.

\section{Tetrabenazine for the Treatment of Chorea}

\author{
Joseph Jankovic, Christine Hunter, \\ and Kevin Dat Vuong. \\ Baylor College of Medicine, Houston, Texas
}

Objective: To describe long-term experience with tetrabenazine (TBZ) in the treatment of chorea.

Background: Dopamine receptor-blocking drugs, commonly used in the treatment of involuntary movements, may cause potentially serious adverse effects, including tardive dyskinesia (TD). This potential serious side effect of dopamine receptor-blocking drugs (neuroleptics) has not been reported with TBZ, a synthetic benzoquinolizine that acts as a monoamine-depleting drug by inhibiting central vesicular monoamine transporter type 2 (VMAT2). TBZ has been reported to have an ameliorating effect in a variety of hyperkinetic movement disorders, including TD, Huntington disease (HD), Tourette syndrome, and other involuntary movement disorders, but the drug has not yet been approved for the treatment of these disorders in the United States. Marked improvement in chorea was reported in $83 \%$ of the first 90 patients, treated between 1980 and $1995 .{ }^{1}$ The drug was well tolerated and no serious adverse events were noted.

Design/Methods: A retrospective chart review was performed on subjects treated with TBZ between December 1996 and January 2004. Response to treatment was assessed by a previously published response scale $(1=$ marked improvement; $4=$ no response; $5=$ worsening). ${ }^{1}$ All adverse events were captured and coded according to their relationship to the study drug.

Results: To date, 165 patients (67 males) with chorea have been treated with TBZ at the Parkinson's Disease Center and Movement Disorders Clinic, Baylor College of Medicine. Of the 98 patients (age, $53 \pm 19$ years; range, 3-80) with evalu-
TABLE 1.

\begin{tabular}{|c|c|c|c|c|c|c|c|}
\hline \multirow[b]{2}{*}{ Disorder } & \multirow[b]{2}{*}{$n$} & \multirow{2}{*}{$\begin{array}{l}\text { Percentage of } \\
\text { Patients with } \\
\text { Response } \\
\text { Rating* } \\
\text { of } 1 \text { or } 2\end{array}$} & \multicolumn{5}{|c|}{$\begin{array}{c}\text { Initial Response } \\
\text { Rating }\end{array}$} \\
\hline & & & 1 & & 3 & 4 & 5 \\
\hline Huntington Disease & 63 & $86 \%$ & 37 & 17 & 7 & 2 & 0 \\
\hline Chorea & 27 & $81 \%$ & 16 & 6 & 2 & 2 & 1 \\
\hline
\end{tabular}

$* 1=$ marked reduction in abnormal movements, excellent improvement in function; 2 = moderate reduction in abnormal movements, very good improvement in function.

able visits since 1997, 68 had HD and the remainder had other choreas. The vast majority of the patients experienced robust improvement in their chorea (Table 1) $(n=90)$. The most frequent adverse effects, all of which were dose-related and none were permanent, included drowsiness or fatigue, depression, and akathisia. No patient experienced orthostatic hypotension or tardive dyskinesia.

Conclusion: TBZ is a safe and effective drug for chronic treatment of chorea associated with HD or other disorders.

\section{REFERENCES}

1. Jankovic J, Beach J. Long-term effects of tetrabenazine in hyperkinetic movement disorders. Neurology 48:358-362, 1997.

\section{New Application Forms of Lisuride, A Potent Dopamine Agonist R. Horowski, ${ }^{*}$ H. Beneš, ${ }^{\dagger}$ D. Woitalla, ${ }^{\ddagger}$ H. Przuntek,
and J. Tack* \\ *NeuroBiotec Berlin; ${ }^{\dagger}$ LKA Schwerin Schlaflabor; ${ }^{\ddagger}$ Josefskrankenhaus Ruhr-Universität Bochum, Germany}

Lisuride, a strong dopamine agonist with high affinities for other monoamine receptors, has been approved in European countries in its oral form for the therapy of Parkinson's disease (PD) and hyperprolactinemia. Prospective long-term studies for up to 10 years have also shown this drug to postpone the development of levodopa long-term complications and to improve the patients' situation. Its very low effective dose as well as its physicochemistry and kinetics make it an ideal candidate for new ways of application, and indeed, Stocchi et al. ${ }^{1}$ have obtained in a 4-year study excellent results when using lisuride in patients with advanced PD for continuous dopaminergic stimulation (CDS) by controlled subcutaneous infusion (12-16 $\mathrm{h}$ /day) using micropumps. The same application form has also caused significant improvement in the therapeutic range in PD as shown by standardized intravenous levodopa challenges. ${ }^{2}$ For easier use for CDS a special transdermal form (NBt P101D) has been developed which, after a lag phase, achieves therapeutic lisuride plasma levels $(200-300 \mathrm{pg} / \mathrm{ml})$ for $48 \mathrm{~h}$. When given every other day, this patch has been shown in early dose-finding studies to exert a clear anti-PD effect in advanced disease as well as significant efficacy in severe restless leg syndrome with additional daytime symptoms. Large multicenter studies have now been started or are in preparation to confirm the therapeutic potential of this new therapy; furthermore, the availability of short-lasting (i.v./s.c. bolus/p.o.) and long-lasting (subcutaneous infusion, transdermal patch) forms of the same drug (lisuride) will become a useful tool for studying possible correlations between pharmacokinetics, effects and 
side effects in PD and other diseases, and will also, hopefully, lead to better, controlled individualized therapy.

\section{REFERENCES}

1. Stocchi F, Ruggieri S, Vacca L, Olanow CW. Prospective randomized trial of lisuride infusion versus oral levodopa in patients with Parkinson's disease. Brain 125:2058-2066, 2002.

2. Baronti F, Mouradian MM, Davis TL, Giuffra M, Brughitta G, Conant KE, Chase TN. Continuous lisuride effects on central dopaminergic mechanisms in Parkinson's disease. Ann Neurol 32: 776-781, 1992.

\section{Imprinted VMAT2 Haplotypes Contribute to Risk for Narcolepsy: Implications for Individual Difference}

\section{George Uhl \\ National Institute on Drug Abuse, Baltimore, Maryland}

Narcolepsy is a familial and heritable sleep disorder with excessive daytime somnolence, abnormal rapid eye movement sleep and often cataplexy, sleep paralysis, or hypnogogic hallucinations. Brain monoamines are implicated by evidence that includes the ability of monoamine-altering stimulant drugs to treat narcoleptic symptoms. The vesicular monoamine transporter VMAT2 (SLC18A2), is thus a candidate to harbor variations that might play roles in narcolepsy. The human VMAT2 gene harbors "B" haplotypes which are preferentially not transmitted from mothers to narcoleptic offspring. "B" haplotype mRNAs are expressed at different levels than "A" haplotye mRNAs in vitro and in vivo. VMAT2 5' flanking/promoter sequences $\mathrm{CpG}$ dinucleotides that lie in and around a USF1 recognition site are methylated. "B" haplotype sites are methylated more strongly and in bimodal patterns not seen in "A" haplotypes. Each of these results supports roles for imprinted VMAT2 haplotypes in a human disorder. Since amphetamines, tetrabenazine, and other therapeutically important drugs act at the monoaminergic vesicles that express VMAT2, these findings suggest that individual differences in responses to these drugs are likely to arise from these VMAT2 haplotypes as well.

\section{From Bench to Bedside: The Journey of $\mathrm{R}(+)$ Pramipexole from Backshelf to Amyotrophic Lateral Sclerosis Clinical Research}

\author{
James P. Bennett, Jr. \\ Center for the Study of Neurodegenerative Diseases, \\ Department of Neurology, University of Virginia, \\ Charlottesville, Virginia
}

Amyotrophic lateral sclerosis (ALS) is a relatively rare neurodegenerative disease of adults that arises from death of upper and lower motor neurons. It is rapidly progressive, with a median survival of 32 months from symptom onset and 19 months from diagnosis. ${ }^{1}$ Small increases in survival, usually no more than a few weeks, have been achieved with riluzole, but ALS remains fundamentally an untreatable and universally fatal disease.

$\mathrm{R}(+)$ pramipexole (PPX) is the enantiomer of S(-) PPX that is a potent D2 dopamine receptor (D2R) agonist marketed as Mirapex for the treatment of Parkinson's disease motor symptoms. Both compounds are antioxidants, with $\mathrm{R}(+)$ PPX having $<1 \%$ of the $\mathrm{D} 2 \mathrm{R}$ receptor potency compared to $\mathrm{S}(-)$, suggesting that it should be tolerated in much higher doses. Beginning in 1997, we initiated cell culture-based studies of the potential usefulness of $\mathrm{R}(+)$ PPX as a neuroprotective com- pound. We found that $\mathrm{R}(+)$ PPX blocked activation of cell death pathways in human SY5Y neuroblastoma cells exposed to methylpyridinium $\left(\mathrm{MPP}^{+}\right)$or $\beta$-amyloid peptides. ${ }^{2} \mathrm{R}(+)$ and S(-) PPX were about equipotent at nanomolar concentrations in inhibiting $\mathrm{MPP}^{+}$-induced cell death. ${ }^{2}$ Mice receiving daily doses of up to $100 \mathrm{mg} / \mathrm{kg} /$ day of $\mathrm{R}(+)$ PPX for 8 weeks showed no motor abnormalities, signs of illness, or histological changes in organs. $\mathrm{R}(+)$ PPX administration to mice blocked MPTP-induced increases in forebrain oxygen free radicals.

Based on this encouraging data, we next examined the ability of the clinically available S(-) PPX to reduce systemic oxidative stress in ALS subjects. We found about twice as much oxidative stress in ALS that was reduced by daily S(-) PPX doses of 4.5-6.0 mg. ${ }^{3}$ As a result, an IND was sought and obtained $(60,948)$ to begin phase I testing of oral R(+) PPX. Very recently, $\mathrm{R}(+)$ PPX was shown by others to be active in the SOD1 G93A mutant ALS mouse model. ${ }^{4}$

$\mathrm{R}(+)$ PPX is an antioxidant/neuroprotective compound that may be active in several related neurodegenerative illnesses. It is highly active in vitro and prolongs survival in the aggressive SOD1 mutant ALS mouse model. It is being developed for neuroprotective therapy at the University of Virginia Center for the Study of Neurodegenerative Diseases for ALS.

\section{REFERENCES}

1. del Aguila MA, Longstreth WT Jr, McGuire V et al. Prognosis in amyotrophic lateral sclerosis: a population-based study. Neurology 60:813-819, 2003.

2. Abramova NA, Cassarino DS, Khan SM et al. Inhibition by R(+) or $\mathrm{S}(-)$ pramipexole of caspase activation and cell death induced by methylpyridinium ion or beta amyloid peptide in SH-SY5Y neuroblastoma. J Neurosci Res 67:494-500, 2002.

3. Pattee et al. Amyotroph Lateral Scler Other Motor Neuron Disord 11:1-6, 2003.

4. Schwalenstoecker et al. $14^{\text {th }}$ Intl Symposium on ALS, Milan, November, 2003.

\section{Upregulation of IL-10 in Patients Treated with Oral Tauferon}

L.H. Villarete, ${ }^{*}$ C.P. Liu, ${ }^{*}$ H.L. Weiner, ${ }^{\dagger}$ and M.J. Tong ${ }^{*}$ *Pepgen Corporation, Alameda, California; ${ }^{\dagger}$ Brigham and Women's Hospital, Harvard Medical School, Boston, Massachusetts; ${ }^{\ddagger}$ Huntington Medical Research Institute, Pasadena, California

Oral administration of Tauferon [interferon (IFN)-tau], a novel type I IFN, is effective in preventing experimental allergic encephalomyelitis (EAE). Prevention of EAE correlates with the induction of the Th2 cytokine IL-10 with a concomitant reduction of the Th1 cytokine IFN-g. In this report, we examined the ability of Tauferon to regulate IL-10 and IFN-g production in patients with relapsing-remitting multiple sclerosis (RRMS) and patients with hepatitis $\mathrm{C}(\mathrm{HepC})$ infection by measuring the serum levels of these cytokines before, during, and after oral administration of the drug. Fifteen RRMS patients in a phase IB clinical study were treated with $0.2 \mathrm{mg}, 0.6$ $\mathrm{mg}$, or $1.8 \mathrm{mg}$ Tauferon once per day for 4 weeks (5 patients per group). At least two patients from each group showed an increase in IL-10 in conjunction with either a decrease or a leveling of IFN-g during drug treatment, indicating a favorable response to the drug. However, because of the high variability of the data in all three groups, statistical significance was not reached. In contrast, a higher dosing regimen, longer treatment duration and slightly increased number of patients in the phase IIA hepatitis $\mathrm{C}$ clinical study resulted in significant increase in 
IL-10 production. Eighteen patients with $\mathrm{HCV}$ were dosed with $1 \mathrm{mg}, 3 \mathrm{mg}$, or $9 \mathrm{mg}$ of Tauferon per day for 84 days (6 patients per group). Our analysis found a statistical significant difference among the three dose groups $(p=0.0009)$ and a significant group-by-time interaction $(p=0.001)$, which is clearly seen by the difference in IL-10 response rate among the three groups over the treatment time period followed by a differential decline once dosing was terminated [group $1(1 \mathrm{mg})$ produced a $22 \%$ increase in IL-10 levels from day 1 to day 43; group 2 $(3 \mathrm{mg}$ ) produced a peak response of $114 \%$ by day 29 ; and group $3(9 \mathrm{mg})$ produced a $387 \%$ increase by day 43 with a peak of $484 \%$ by day 71$]$. There was no significant difference between the three dose groups ( $p>0.3499)$, no significant effect of time $(p=0.1187)$, and no significant group-by-time interaction ( $p=$ 0.1275 ) for IFN-g in the same HepC patients, suggesting IFN-g levels were unchanged during Tauferon treatment.

\section{Kynurenic Acid and 7-CL-Kynurenic Acid Production in Epileptic Tissue: Studies in Rats and Humans}

A. Rassoulpour,* H.Q. Wu, ${ }^{*}$ P. Guidetti, ${ }^{*}$ H. E. Scharfman, ${ }^{\dagger}$ G. M. McKhann, ${ }^{\ddagger}$ R. R. Goodman, ${ }^{\ddagger}$ E. H. Bertram, ${ }^{\S}$ and R. Schwarcz*

* Maryland Psychiatric Research Center, University of Maryland School of Medicine, Baltimore, Maryland; ${ }^{\dagger}$ Center for Neural Recovery and Rehabilitation Research, Helen

Hayes Hospital and Columbia University, West Haverstraw, New York; ${ }^{\ddagger}$ Department of Neurosurgery, Columbia University School of Medicine, New York, New York; ${ }^{\S}$ Department of Neurology, University of Virginia, Charlottesville, Virginia

Targeting of the glycine coagonist site of the NMDA receptor is considered a viable strategy to identify effective neuroprotective and anticonvulsant agents. 7-Cl-kynurenic acid (7Cl-KYNA), a synthetic derivative of the glutamate receptor antagonist kynurenic acid (KYNA), is a selective antagonist of this site and shows potent anticonvulsant and neuroprotective properties in various animal models. Both in vivo and in vitro studies have demonstrated the ability of astrocytes to enzymatically produce KYNA [from its bioprecursor kynurenine (KYN)] and 7-Cl-KYNA [from its synthetic precursor 4-Clkynurenine (4-Cl-KYN)]. We evaluated these conversions in tissues from animals exhibiting chronic seizure activity and in surgical tissue from a temporal lobe epilepsy (TLE) patient. Tissue slices from hippocampus and entorhinal cortex (EC) were incubated for $2 \mathrm{~h}$ with $2 \mathrm{~m} \mathrm{KYN}$ and 2 or $20 \mathrm{~m} 4-\mathrm{Cl}-\mathrm{KYN}$, and the release of newly produced KYNA and 7-Cl-KYNA was monitored in the extracellular medium (cf. J Neurochem 69: 290, 1997). KYNA and 7-Cl-KYNA production was increased in chronically epileptic rats compared to controls $(p<0.05)$. KYNA formation in hippocampal and EC tissue from the TLE patient was quantitatively similar to epileptic rat tissue. 7-ClKYNA neosynthesis in human tissue was linear with substrate concentration and, unexpectedly, was fourfold more effective than in rats. These results demonstrate the ability of epileptic rat and human brain tissue to produce KYNA and 7-Cl-KYNA and bode well for the use of 4-Cl-KYN in the treatment of TLE.

This work was supported by United States Public Health Service Grant NS16102. Dr. Schwarcz has a proprietary interest in Artemis Neuroscience, which develops 4-chlorokynurenine for the treatment of temporal lobe epilepsy.

\section{Continuous Apomorphine Administration with Novel Ethylene-Vinyl Acetate Implants Reduces the Risk of Motor Complication}

\author{
Francesco Bibbiani, ${ }^{*}$ Aiste Kielaite, ${ }^{*}$ Lauren Costantini, ${ }^{\dagger}$ \\ and Thomas Chase* \\ * National Institutes of Health/National Institutes of \\ Neurological Disorders and Stroke Experimental \\ Therapeutics Branch, Bethesda, Maryland; ${ }^{\dagger}$ Titan \\ Pharmaceuticals, Inc., South San Francisco, California
}

Objective: To determine whether continuous delivery of apomorphine through ethylene-vinyl acetate (EVA) implants delays the development of motor complications observed after chronic pulsatile administration of dopaminergic agents in treatment-naive parkinsonian primates.

Background: Normal motor function is dependent on the continuous synthesis and release of dopamine by neurons projecting from the nigra to the striatum. In Parkinson's disease, the degeneration of dopaminergic neurons and the pulsatile stimulation provided by usual dopamine replacement therapies appear to contribute to the development of motor complications such as dyskinesias. A subcutaneously implantable polymeric matrix (EVA) has been developed for the sustained systemic delivery of drugs. Preclinical studies showed that this delivery system provides controlled release of several medications for extended periods.

Design/Methods: Four MPTP-lesioned, L-dopa-naive cynomolgus monkeys each received three EVA rods containing apomorphine (98 mg, 10\% per rod). Three additional MPTPlesioned monkeys received daily injections of $0.2 \mathrm{mg} / \mathrm{kg}$ apomorphine (minimally effective dose to turn animal "ON").

Results: All EVA-implanted animals were continuously "ON" within 1 day after implantation. All animals in the pulsatile injection group were "ON" for approximately $90 \mathrm{~min}$ after each administration of apomorphine. After 8.3 days (range 7-10) of daily apomorphine injections, all animals in the pulsatile injection group developed dyskinesias. In the continuous delivery EVA-implanted group, no animal developed dyskinesia for the duration of treatment (up to 6 months). The "ON" state induced by continuous apomorphine delivery was clinically similar to that induced by injection of apomorphine (Laval scale scores, 0-1). The first implanted animal remained "ON" with sustained apomorphine plasma levels for 7 weeks. Local irritation developed beginning 2 days after implant and resolved within 2 weeks. The second animal was pretreated with dexamethasone $(0.5 \mathrm{mg} / \mathrm{kg}) 1$ day before implantation, day of implantation, and 2, 4, and 6 days after. This animal remained "ON" with sustained plasma levels for 24 weeks. Local irritation developed at 22 weeks; implants were removed and histology performed. The third and fourth animals (also pretreated with dexamethasone) have been implanted for 8 weeks, and maintain "ON" state with no signs of local irritation or dyskinesias. Implanted animals maintained "ON" with lower plasma levels of apomorphine when compared with the higher plasma levels required to reach "ON" with pulsatile apomorphine, which leads to dyskinesias.

Conclusions: Apomorphine EVA implants provide continuous plasma levels of apomorphine, allowing continuous stimulation of striatal dopaminergic receptors which appears to prevent onset of dyskinesias for the treatment period, which in this ongoing series of evaluations is up to 6 months. 


\section{The Role of Striatal PKA in the Development of Motor Deficit in an Animal Model of Parkinson's Disease}

Irene Avila, Justin D. Oh, Edward Castañeda, Christopher P.S. Smith, and Thomas N. Chase

Parkinson's disease (PD) results from the degeneration of the nigrostriatal pathway, leading to the depletion of dopamine in the striatum. When dopamine (DA) is lost, it may produce changes in 1) kinase signaling, 2) corticostriatal glutamatergic input to the striatum, and 3) motor output. A preliminary study is being conducted in the 6-hydroxydopamine (6-OHDA) lesioned rat to determine if there is a correlation between motoric response alterations, as determined by skilled reaching using the Montoya staircase box, and cyclic-AMP-dependent protein kinase A (PKA) signaling mechanism in the striatum. Three weeks after dopaminergic denervation in the hemisphere contralateral to the preferred limb, rats demonstrated a decrease in forelimb function, as measured by changes in total number of pellets eaten. No change in the number of pellets eaten was seen with the limb ipsilateral to the lesioned side. Protein levels in the striatum for various isoforms of PKA (PKA: $\alpha, \beta$, and $\gamma$ catalytic subunits; PKA: $\mathrm{I} \alpha, \mathrm{I} \beta, \mathrm{II} \alpha$, and $\mathrm{II} \beta$ regulatory subunits), at least in part, appear to be related to deficits in reaching task performance in these rats. These preliminary observations will provide insight into the possible cellular substrates associated with 6-OHDA lesion-induced extrapyramidal motor impairments. Future plans for this study are to pharmacologically block the kinase signaling and determine its functional implications in the motor behavior.

\section{Enhanced Striatal PKC Lambda Contributes to the Development of Levodopa-Induced Motor Response Alterations in Hemiparkinsonian Rats}

\author{
Christopher P.S. Smith, * Justin D. Oh, ${ }^{\dagger}$ \\ and Thomas N. Chase* \\ *Experimental Therapeutics Branch, National Institutes of \\ Health, Bethesda, Maryland; ${ }^{\dagger}$ Department of Psychology, \\ Central Michigan University, Mount Pleasant, Michigan
}

Levodopa continues to be the most effective treatment for Parkinson's disease (PD), although its chronic administration has been associated with the appearance of disabling motor response complications. Recent evidence suggests that they appear to involve sensitization of ionotrophic glutamate receptors on striatal medium spiny neurons, reflecting, in part, changes in the phosphorylation state of NMDA and AMPA receptor subunits as a result of the aberrant activation of protein kinase $\mathrm{C}$ (PKC). To determine whether PKC activation in striatal spiny neurons contributes to the development of levodopainduced response fluctuations of the "wearing-off" type, we examined the ability of the PKC antagonist tamoxifen to prevent as well as palliate the characteristic shortening in duration of the motor response to acute levodopa challenge in hemiparkinsonian rats given chronic levodopa therapy. Three weeks of twice-daily levodopa treatment shortened the duration of contralateral rotation to levodopa by about $20 \%(p<0.05)$. At the same time, protein expression of an atypical PKC isoform, PKC lambda, increased ipsilateral to the lesion $(34 \pm 6 \%$ of intact striatum in levodopa-treated animals, $p<0.05$ compared with controls). Pharmacological blockade of PKC with tamoxifen $(5.0 \mathrm{mg} / \mathrm{kg}$, p.o.), coadministered twice daily with levodopa, diminished the levodopa-induced truncation of response duration $(p<0.05)$. The single-dose administration of tamox- ifen $(5$ or $10 \mathrm{mg} / \mathrm{kg}$, p.o.) 1 hour before levodopa injection also reversed the response shortening $(p<0.05)$. These results suggest that augmented PKC lambda, alone or together with striatal glutamate receptor sensitization, contributes to the apparent alterations in striatal neuronal function and thus to the plastic modifications in dopaminergic responses in hemiparkinsonian rats.

Key Words: Parkinson, 6-OHDA, tamoxifen, striatum.

This work was supported by the National Institute of Neurological Disorders and Stroke.

\section{Markers of Neurodegenerative Diseases in Cerebrospinal Fluid}

Xiaoxia Wang, Gerda Andringa, William Bara-Jimenez,

Emory Encarnacio, Michael Morris, Amy Bridgeman, Thomas Chase, and Catherine Bennett

Experimental Therapeutics Branch, National Institute of Neurological Disorders and Stroke, Bethesda, Maryland; Blanchette Rockefeller Neurosciences Institute, Morgantown, West Virginia

Increased tissue transglutaminase (tTG) and tTG-crosslinked protein have been reported in Alzheimer's disease (AD) and Parkinson's disease (PD) brain and in CSF of AD patients. Furthermore, AD- and PD-associated proteins tau, $\beta$-amyloid and $\alpha$-synuclein are substrates of tTG. The identification of protein markers in human CSF for neurodegenerative diseases provides the dual possibility of 1) affording more definitive early diagnoses using peripheral biomarkers, and 2) identifying pathological processes that may point to therapeutic intervention. To examine human CSF for tTG-crosslinked proteins, CSF samples were obtained by lumbar puncture from patients with $\mathrm{AD}(n=4), \mathrm{PD}(\mathrm{n}=6)$ and multiple system atrophy (MSA; $\mathrm{n}=1$ ). Samples were analyzed to detect $\alpha$-synuclein and proteins containing the $\mathrm{N}^{\epsilon}$ ( $\tau$-glutamyl) lysine isodipeptide, which characterizes the tTG-induced covalent bond, using Western immunoblot. We identified a tTG-crosslinked protein of approximately $52 \mathrm{kDa}$ present in all $\mathrm{AD}$ samples, and in higher concentration in the MSA sample, but in none of the PD samples. The identification of this protein may indicate a novel pathology in AD and MSA. Three oligomers containing $\alpha$-synuclein were detected in samples from all patients; one protein migrating at approximately $28 \mathrm{kDa}$ and the other two in lesser concentration at approximately 52 and $60 \mathrm{kDa}$. The $\alpha$-synuclein monomer appeared only in one sample that visibly contained red blood cells. These data indicate the presence of $\alpha$-synuclein oligomers in CSF; however, further testing is required to determine whether this feature is pathological.

\section{Minocycline in Huntington's Disease: A Pilot Study}

Madhavi Thomas, ${ }^{*}$ Joseph Jankovic, ${ }^{\dagger}$ and Tetsuo Ashizawa ${ }^{*}$ *Experimental Therapeutics Branch, National Institute of Neurological Disorders and Stroke, Bethesda, Maryland; ${ }^{\dagger}$ Baylor College of Medicine, Houston, Texas; ${ }^{\sharp}$ University of Texas Medical Branch, Galveston, Texas

Aim: To study the tolerability and safety of minocycline in patients with Huntington's disease (HD).

Background: Minocycline is a caspase inhibitor, decreases inducible nitric oxide synthase (iNOS), and has been shown to delay disease progression in the mouse model R6/2 of HD.

Methods: This safety and tolerability study included 30 patients with HD who were given minocycline over a 6-month 
period and underwent assessments every 2 months with laboratory studies, the abnormal involuntary movements scale, the unified Huntington's disease rating scale, and the mini-mental state examination.

Results: Minocycline was well tolerated during this study period and no serious adverse events were noted.

\section{Catechol- $O$-Methyltransferase val108/158met Genotype Predicts Working Memory Response to Antipsychotic Medication}

Thomas Weickert, Terry Goldberg, Aaron Mishara, Jose Apud, Bhaskar Kolachana, Michael Egan, and Daniel Weinberger

National Institute of Mental Health, Bethesda, Maryland

The gene encoding catechol- $O$-methyltransferase (COMT), an enzyme that regulates prefrontal cortex dopamine, contains a common functional polymorphism (val ${ }^{108 / 158}$ met) that influences prefrontal cortex function in an allelic dose-dependent manner. A previous study has reported that COMT val-met genotype also influences the cognitive response to treatment with a second-generation antipsychotic medication, which has also been associated with prefrontal cortex function. We tested the effect of COMT genotype on the cognitive response to antipsychotic medications in patients with schizophrenia using a test of working memory previously shown to be sensitive to COMT genotype. Twenty inpatients ( 5 with val-val genotype, 11 val-met, and 4 met-met) with schizophrenia or schizoaffective disorder were administered the n-back working memory test at two time points: once following 4 weeks of treatment with antipsychotic medication and once following 4 weeks of placebo administration, in a counterbalanced, double-blind, within-subject design. Patients with met-met genotypes displayed significant improvement on the working memory task during treatment. COMT val-val patients did not improve on this task and displayed continuing working memory deficits. These results support other data that COMT val ${ }^{108 / 158}$ met genotype may be an important factor in the cognitive response to antipsychotic medication. 